\title{
Análisis de armónicas dinámicas con filtros de respuesta impulsional finita diseñados con 0-splines
}

\author{
José Antonio De la O Serna \\ Universidad Autónoma de Nuevo León, \\ Facultad de Ingeniería Mecánica y Eléctrica \\ jose.delaosr@uanl.edu.mx
}

\section{RESUMEN}

Los splines son esenciales en procesamiento de señales. No solamente se usan en el muestreo e interpolación de señales, sino también en el diseño de filtros, en procesamiento de imágenes, y el análisis multiresolución. Aqui presentamos una nueva clase de splines. Se les llama O-splines porque sus nodos están separados por un ciclo fundamental. Se usan como muestreadores de estados óptimos, en el sentido de que sus coeficientes ofrecen en cada instante de tiempo las derivadas del segmento de señal que aseguran la mejor aproximación de Taylor alrededor de un punto, o la mejor interpolación de Hermite entre dos puntos. Los $O$-splines corresponden a la respuesta impulsional de los filtros de la Transformada de Tiempo Discreto de Taylor-Fourier (DTTFT). Los pasabajas coinciden con los núcleos centrales de interpolación de Lagrange, los cuáles convergen a la función Seno Cardenal, respuesta impulsional del filtro ideal. Y sus derivadas convergen a su vez a los diferenciadores ideales pasabajas. Los $O$-splines pasabanda son splines armónicos, pues son simples modulaciones de un pasabajas en cada frecuencia armónica. En este artículo se presenta la solución en forma cerrada de los $O$-splines pasabajas. Con ella se reduce enormemente la complejidad computacional de la DTTFT y se pueden obtener O-splines de orden elevado. Con ellos se pueden diseñar filtros pasabanda muy cercanos al ideal, en cualquier frecuencia. Los $O$-splines definen una escalera de espacios muy útiles para el análisis multiresolución, y el análisis tiempo-frecuencia. El artículo ilustra algunos ejemplos de diversa naturaleza. Por supuesto que una nueva familia de onduletas obtenidas a partir de los O-splines está en camino.

\section{PALABRAS CLAVE}

Splines cardenales, núcleo de interpolación central de Lagrange, Transformada de tiempo discreto Taylor-Fourier, señales oscilatorias, oscilaciones de potencia, análisis tiempo-frecuencia, análisis multiresolución, compresión de datos.

\section{ABSTRACT}

Splines are at the essence of signal processing. Not only in sampling and interpolation, but also in filter design, image processing, and multi-resolution analysis. A new class of splines is presented here. They are referred to as $O$ splines since their knots are separated by one fundamental cycle. They are used as optimal state samplers, in the sense that their coefficients provide the 
derivatives for the best Taylor approximation to a given signal about a time instance or the best Hermite interpolation between two of them. They are the impulse response of the filters of the Discrete-Time Taylor-Fourier Transform (DTTFT) filter bank. Lowpass O-spline coincides with the Lagrange central interpolation kernel, which converges towards the ideal Sinc function. It comes with its derivatives which in turn converge to the ideal lowpass differentiator. The bandpass $O$-splines are harmonic splines since they are modulations of the former kernel at harmonic frequencies. In closed-form, they reduce the computational complexity of the DTTFT and can be used to design ideal bandpass filters at a particular frequency. By increasing the order they define a ladder of spaces very useful for multi-resolution and time-frequency analysis. Examples are provided at the end of the paper. Naturally, a new family of wavelets is coming soon from these splines.

\section{KEYWORDS}

Cardinal splines, central Lagrange interpolation kernel, Taylor-Fourier discrete time transform, oscillatory signals, power oscillations, time-frequency analysis, multi-resolution analysis, data compression.

\section{INTRODUCCIÓN}

Los splines ${ }^{1,2}$ están no solamente en la base de las operaciones del procesamiento de señales, tales como el muestreo y la interpolación, sino también en el diseño de filtros, ${ }^{3}$ el procesamiento de imágenes, ${ }^{4}$ y el análisis multi-resolución. ${ }^{5}$ Ellos se han aplicado igualmente en el diseño asistido por computadora, en computación gráfica para dibujar curvas suaves con curvatura mínima, y para reproducir modelos gráficos y representación de superficies.

Los splines más conocidos en procesamiento de señales son los B-splines.6-7 Lo B-splines de orden $\mathrm{m}$ son continuos hasta la derivada $\mathrm{m}$ - 1. Esta propiedad garantiza juntas continuas. Tienen soporte compacto, pero no son spline cardenales. Sin embargo, ellos se encuentran en la base del marco matemático para el diseño de los splines cardenales de interpolación modernos, tales como los llamados B-splines exponenciales, o E-splines, ${ }^{8,9}$ o combinaciones lineales de ellos.

En esta investigación se obtuvo una nueva clase de splines. Los O-splines fueron obtenidos numéricamente en ${ }^{10}$ para analizar señales oscilatorias de sistemas de potencia. También han sido usados para analizar electro-encefalogramas. ${ }^{11}$ Pero aquí se obtienen en forma cerrada para diseñar filtros pasa-banda y reducir la carga computacional en el análisis de señales de banda limitada.

Los O-splines son las respuestas impulsionales del banco de filtros de la transformada de tiempo discreto Taylor-Fourier (DTTFT).$^{10}$ Ellos son las funciones duales de las del modelo de señal Taylor-Fourier, el cual no es más que la extensión de Taylor de la transformada de Fourier discreta (DFT), en el cual los coeficientes de Fourier se extienden a polinomios de Taylor. Se llaman O-splines porque sus segmentos son cíclicos, es decir sus nudos se separan en intervalos de un ciclo fundamental, y porque ofrecen coeficientes óptimos para la representación de las señales en el subespacio Taylor-Fourier.

Los O-splines en forma cerrada se obtienen mediante la factorización del 
modelo de señal DTTFT en dos operadores: uno para los términos de Taylor, y el otro con las exponenciales complejas de Fourier en las frecuencias armónicas. En esta descomposición, los O-splines y sus derivadas se encuentran en los vectores de la matriz dual del operador de Taylor, y los O-splines pasabanda son simple modulaciones a las frecuencias armónicas de la matriz de Fourier.

Los O-splines pasabajas resultan ser los kernels de interpolación central de Lagrange, los cuáles son de soporte compacto y convergen a la función Seno Cardenal Sinc cuando el orden tiende a infinito. ${ }^{12}$ En consecuencia su respuesta en frecuencia converge a la del filtro pasabajas ideal, y las de sus derivadas a las respuestas en frecuencia de los diferenciadores ideales.

Como los O-splines operan en la etapa de análisis en la matriz dual, ${ }^{13}$ funcionan como muestreadores de estados de la señal, es decir en cada instante de tiempo ofrecen muestras de la señal y de sus primeras derivadas, ofreciendo los coeficientes óptimos para la mejor aproximación de Taylor a la señal en la ecuación de síntesis. Esta es una diferencia importante con respecto a la mayoría de los splines, cuya literatura se consagra a su rendimiento como interpoladores. ${ }^{14}$ Esta característica distingue este trabajo de investigación de los encontrados en la literatura.

Los trabajos más recientes de splines cardenales de interpolación son sobre los splines de muchos nódulos MK-splines. ${ }^{15}$ Éstos son filtros FIR simétricos con muestras en cada mitad de intervalo y obtenidos por combinaciones lineales de Bsplines del mismo orden. Tienen dos trozos polinomiales por intervalo unitario con el doble de nudos (de ahí su nombre). Aun cuando MK-y O-splines tienen soporte compacto, los O-splines no tienen nada que ver con los B-splines. Su cardenalidad resulta directamente en el operador adjunto de la expansión Taylor-Fourier. En consecuencia, los O-splines son aplicados como operadores de muestreo, operación inversa a la de interpolación. Ellos obtienen los mejores coeficientes Taylor-Fourier, con significado físico de posición, velocidad y aceleración de la señal. Al estar en la base dual de un sistema biortogonal, establecen un proceso híbrido de análisis-síntesis. Estas son las principales distinciones de los O-splines, que los hacen únicos, óptimos, útiles, efectivos y eficaces.

Recientemente, los MK-splines fueron generalizados a los GMK-splines, ${ }^{16}$ una familia de splines obtenidos mediante la relajación a racionales de la mitad de intervalo de los MK. Aun cuando esta relajación produce algunos filtros con ganancias de paso no-planas, y otros con soporte fraccionario (no entero), los GMK splines de orden $\mathrm{n}$ tienen continuidad de orden $\mathrm{n}-1$.

El modelo de señal DTTFT se concibió como una extensión del tradicional concepto de fasor (coeficiente de Fourier) al de fasor dinámico para capturar oscilaciones con mejor exactitud. Este modelo extendido corresponde al de señales en el $\operatorname{span}\left\{t^{n} e^{j 2 \pi h F_{1} t}\right\}_{h=-\frac{N}{2}, \ldots, \frac{N}{2}-1 ; n=0, \ldots, K}$ donde $F_{1}$ is la frecuencia fundamental, y $\mathrm{N}$ es el número de armónicas dentro de la banda delimitada por la frecuencia de muestreo. Esto corresponde al emphspan del conjunto de polos con multiplicidad $K+1$ en las $N$ frecuencias armónicas. ${ }^{9}$ Bajo este aspecto, los $\mathrm{O}$-splines son splines exponenciales con argumentos imaginarios, o splines de Fourier y cumplen con las propiedades y teoremas de los B-exponenciales en. ${ }^{8}$

La ecuación de síntesis de la DTTFT interpola un polinomio de Taylor del 
orden del spline, sobre el intervalo del spline, pero con las derivadas estimadas también es posible hacer una interpolación de Hermite entre dos estados cíclicos consecutivos. El muestreador de estados dotado con un interpolador de Hermite ofrece un algoritmo de compresión óptima de señales oscilatorias y reduce el costo computacional con un error suficientemente pequeño controlado por el orden de los O-splines a la entrada y el grado del spline de Hermite a la salida.

En lo que sigue, el modelo de señal DTTFT se presenta en la Sección II. Los O-splines pasabajas se obtienen en forma cerrada en la Sección III, junto con sus derivadas y sus respuestas en frecuencia. Enseguida, los O-splines armónicos se establecen en la Sección mbox III-D mbox . Como resultados IV se usan los O-splines para analizar y reconstruir una senoidal, para eliminar el ruido de una oscilación de frecuencia variable de un sistema real de potencia, ${ }^{17,}{ }^{18}$ y para separar los modos de oscilación de una forma de onda de presión sanguínea de un paciente. ${ }^{19}$ Finalmente se presentan las conclusiones del artículo.

\section{TRANSFORMADA DE TIEMPO DISCRETO TAYLOR-FOURIER}

Los O-splines de la DTTFT O-splines fueron presentados en ${ }^{10}$ en forma matricial. En el presente artículo se obtienen en forma cerrada. Esta formulación reduce la complejidad computacional y ofrece una secuencia de funciones cuyos espectros convergen al del filtro pasabajas ideal.

El modelo de señal Taylor-Fourier se obtiene relajando los coeficientes de Fourier a funciones temporales polinomiales de la forma, $\xi_{h}(t)=a_{h}(t) e^{j \varphi_{h}(t)}$, las cuales portan la envolvente compleja de cada frecuencia armónica h. Así, el modelo de señal extendido se expresa de la siguiente forma:

$$
x(t)-\sum_{h=\infty}^{\infty} \xi_{h}(t) e^{22 \pi h r_{1} t}, \quad-\frac{C T_{1}}{2} \leq l \leq \frac{C T_{1}}{2}
$$

donde $\mathrm{F} 1$ es la frecuencia fundamental en $H z, T 1$ es el período $\left(T_{1}=\frac{1}{F_{1}}\right)$ en segundos, y $C$ es el númbero de ciclos.

De esta forma, las armónicas se convierten en señales de banda angosta. ${ }^{20} \mathrm{La}$ envolvente compleja $\xi_{h}(t)$ en cada frecuencia armónica es entonces aproximada por el mejor polinomio de Taylor de grado $K$ :

$$
\xi_{h}^{(K)}(t)=\xi_{h}\left(t_{0}\right)+\dot{\xi}_{h}\left(t_{0}\right)\left(t-t_{0}\right)+\cdots+\xi_{h}^{(K)}\left(t_{0}\right) \frac{\left(t-t_{0}\right)^{K}}{K !},
$$

en el sentido óptimo de mínimos cuadrados (LMS), en cada intervalo de observación de $C$ ciclos, y centrado en el instante $t_{0}$.

Al tomar $N$ muestras por período fundamental, la ecuación de síntesis del subespacio DTTFT puede escribirse de (1) y (2) como sigue:

$$
-\left(1\left(\begin{array}{c}
W_{N} \\
W_{N} \\
\vdots \\
W_{N}
\end{array}\right) \quad T\left(\begin{array}{c}
W_{N} \\
W_{N} \\
\vdots \\
W_{N}
\end{array}\right) \quad \cdots \frac{1}{k !} T^{K}\left(\begin{array}{c}
W_{N} \\
W_{N} \\
\vdots \\
W_{N}
\end{array}\right)\right)\left(\begin{array}{c}
\xi_{N} \\
\xi_{N} \\
\vdots \\
K_{k} \\
\xi
\end{array}\right)
$$

donde la matriz $\Phi(K+1) N \times(K+1) N$ contiene los vectores de la base vectorial del subespacio, y los subvectores $\xi_{N}^{(k)}, k=0,1, \ldots K$, contienen las derivadas de los fasores dinámicos de cada armónica $\xi_{N}(h)$ en (2). La submatriz $W_{N} N$ $\times N$ es la matriz de Fourier de la transformada de Fourier Discreta (DFT) con columnas armónicas $w_{h}=e^{j \frac{2 \pi}{N} h n} ; h, n=0, \ldots, N-1$. Y finalmente la matriz diagonal 
$T(K+1) N \times(K+1) N$ contiene las muestras del primer término de Taylor en el intervalo de $C=K+1$ ciclos fundamentales. $\operatorname{Ver}^{10}$ para más detalles de esta formulación.

Note en (3) que aumentando el orden de Taylor por uno, el tamaño del modelo aumenta por $N$, ya que cada término de Taylor afecta al conjunto de armónicas, y por tanto se requiere otro ciclo fundamental de datos al fondo dela matriz $\Phi$. Note también que las matrices diagonales de Taylor (y sus potencias), operan productos Hadamard de los segmentos cíclicos en sus diagonales con las columnas de las matrices $W_{N}$.

En, ${ }^{10}$ se demostró que la matriz $\Phi$ puede factorizarse en dos:

$$
\begin{aligned}
\Phi & =\Upsilon \Omega 2 \\
& =\left(\begin{array}{cccc}
I & Y_{1} & \ldots & \frac{1}{1} Y_{1}^{K} \\
I & Y_{2} & \ldots & \frac{1}{K !} Y_{2}^{K} \\
\vdots & \vdots & \ddots & \vdots \\
I & Y_{U} & \ldots & \frac{1}{K !} Y_{C}^{K}
\end{array}\right)\left(\begin{array}{cccc}
W_{N} & 0 & \ldots & 0 \\
0 & W_{N} & \ldots & 0 \\
\vdots & \vdots & \ddots & \vdots \\
0 & 0 & \ldots & W_{N}
\end{array}\right),
\end{aligned}
$$

Esta factorización es posible gracias a que las columnas de $W_{N}$ contienen un período fundamental. Las submatrices diagonales $Y_{c}, c=1,2, \ldots, C, N \times N$, contienen muestras de segmentos cíclicos consecutivos de la matriz de Taylor $T$ in (3). El producto Hadamard en (3) se obtiene en (4) bloque por bloque, de cada columna de submatrices en $\Upsilon$, con las submatrices $W_{N}$ en la diagonal de $\Omega$, reconstruyendo las submatrices verticales de (3).

La siguiente ecuaicón ofrece los mejores coeficientes (Least Mean Squares, LMS) de la proyección de $x$ sobre el subespacio Taylor-Fourier:

$$
\widehat{\xi}=\widetilde{\Phi}^{H} x
$$

en la cual $\widetilde{\Phi}$ es la matriz dual de $\Phi,{ }^{21}$ dada por

$$
\widetilde{\Phi}=\Phi\left(\Phi^{H} \Phi\right)^{-1} \text {. }
$$

$\mathrm{Y}$ con $\Phi=\Upsilon \Omega$ en (4), se tiene:

$$
\widetilde{\Phi}=\Upsilon\left(\Upsilon^{H} \Upsilon\right)^{-1} \frac{\Omega}{N}=\widetilde{\Upsilon} \frac{\Omega}{N},
$$

lo que indica que las columnas de $\widetilde{\Phi}$ son modulaciones a las frecuencias armónicas de las columnas de submatrices diagonales en $\widetilde{\Upsilon}$, es decir, las columnas de submatrices diagonales en $\widetilde{\Upsilon}$ contienen las envolventes comunes (el O-spline y sus derivadas) de cada frecuencia armónica en WN.

Ya que $\Upsilon$ es una matriz cuadrada invertible, su dual es simplemente la transpuesta de su inversa:

$$
\widetilde{\Upsilon}=\Upsilon^{-T}=\frac{\operatorname{Adj}(\Upsilon)^{T}}{|\Upsilon|}
$$

la cual es simplemente la matriz de cofactores $\operatorname{Adj}(\Upsilon)^{T}$ dividida por el determinante de $Y$.

La multiplicación de submatrices diagonales $Y_{i}^{k}$ en el operador de Taylor $\Upsilon$ produce el producto punto a punto de sus diagonales. En consecuencia, $\Upsilon$ se puede compactar en una matriz $C \times C$ sustituyendo cada submatriz $Y_{i}^{k}$ por el $k$-ésimo término de Taylor como una función de tiempo evaluada en su correspondiente iésimo segmento. Así se puede obtener fácilmente la inversa de esa matriz compacta ya que esas funciones actúan como cofactores en la inversión.

En la siguiente sección se obtienen en forma cerrada los O-splines pasabajas de orden $K$ y sus primeras derivadas de el numerador en (7) cuando cada 
submatriz WN en $\Omega$ se reduce a su primer vector columna de unos. Se denota con 0 la submatriz resultante, ya que esa operación convierte las diagonales en cada renglón de en un vector que concatena dichas diagonales en un polinomio a trozos, es decir, en un spline. Las diagonales en las submatrices del primer renglón ofrecen el O-spline pasabajas, y las de los renglones subsecuentes en sus primeras derivadas. Los O-splines armónicos o pasabanda serán simplemente modulaciones de los pasabajas en cada frecuencia armónica.

\section{O-SPLINES PASABAJAS EN FORMA CERRADA}

En las siguientes subsecciones se obtienen los O-splines pasabajas y sus primeras derivadas en 0 mediante la solución en forma cerrada de la matriz inversa de $\Upsilon$ en (8) y concatenando los pedazos polinomios de cada vector de 0 .

Para $K=0$, se tiene: $t_{1}=t_{\left[-\frac{T_{1}}{2}, \frac{T_{1}}{2}\right)}$ y $\Phi_{0}^{(0)}=1$, y por tanto $\widetilde{\Phi}_{0}^{(0)}=1$. El sistema es ortogonal y el O-spline es el pulso rectangular.

$$
\hat{\varphi}_{0}^{(0)}(l)= \begin{cases}1 & -\frac{T_{1}}{2}<1<\frac{T_{1}}{2} \\ 0 & \text { otherwise. }\end{cases}
$$

Para $K=1, t_{1}=t_{\left[-T_{1}, 0\right)}$, y $t_{2}=t_{[0, T 1)}=t_{1}+T_{1}$, tenemos

$$
\Phi_{0}^{(1)}=\left(\begin{array}{cc}
1 & t_{1} \\
1 & t_{2}
\end{array}\right)
$$

$\operatorname{con}|\Phi 0(1)|=t_{2}-t_{1}=T_{1}$. Entonces se tiene:

$$
\Phi_{i !}^{(1)}-\frac{\left(\begin{array}{cc}
l_{2} & -1 \\
-l_{1} & 1
\end{array}\right)}{T_{1}}-\left(\begin{array}{cc}
u_{1}-1 & -F_{1} \\
-\left(u_{2}-1\right) & +t_{1}^{1}
\end{array}\right)
$$

donde $u_{n}$ es el tiempo normalizado $u=t_{h} / T_{1}$. Sus columnas forman el pulso rectangular:

$$
\varphi_{0}^{(1)}(u)= \begin{cases}u+1 & \text { for } \quad-1 \leq u<0 \\ 1-u & \text { for } 0 \leq u<1 \\ 0 & \text { otherwise }\end{cases}
$$

y la onduleta de Haar: $-F_{1} \dot{\widetilde{\varphi}}_{0}^{(1)}(u)$.

Para $K=2$, tenemos

$$
\Phi_{0}^{(2)}-\left(\begin{array}{ccc}
1 & t_{1} & t_{1}^{2} / 2 \\
1 & t_{2} & t_{2}^{2} / 2 \\
1 & t_{3} & t_{3}^{2} / 2
\end{array}\right)
$$

$$
\begin{aligned}
\text { con } t_{1} & =t_{\left[-\frac{3 T_{1}}{2},-\frac{T_{1}}{2},\right.}, t_{2}=t_{1}+T_{1}, \mathrm{y} t_{3}=t_{1}+2 T_{1} \text {. En este caso }\left|\Phi_{0}^{(2)}\right|=T_{1}^{3}, \mathbf{y} \\
\tilde{\Phi}_{0}^{(2)} & =\left(\begin{array}{crc}
\frac{1}{2}\left(u_{1}-2\right)\left(u_{1}+1\right) & -F_{1}\left(u_{1}-\frac{3}{2}\right) & F_{1}^{2} \\
-\left(u_{2}+1\right)\left(u_{2}-1\right) & 2 F_{1} u_{2} & -2 F_{1}^{2} \\
\frac{1}{2}\left(u_{3}-1\right)\left(u_{3}-2\right) & -F_{1}\left(u_{3}-\frac{3}{2}\right) & F_{1}^{2}
\end{array}\right) .
\end{aligned}
$$

Note en (11) y (14), que es suficiente con calcluar la primera columna de la matriz dual, ya que las siguientes son el negativo de la derivada de la precedente escalada por factores constantes. De hecho, como el primer vector columna es simétrico, solamente se requiere su primera mitad para obtener todos los vectores restantes.

De (13) se puede ver que $\Phi 0(\mathrm{~K})$ es una matriz de Vandermonde con columnas escaladas. En el Apéndice A se comprueba que su determinante es $\left|\Phi_{0}^{(K)}\right|=T_{1}^{\frac{K(K+1)}{2}}$. 
Finalmente, para

$K-3, l_{1}-t_{\left.-2 T,-T_{1}\right)} \mathrm{y} t_{n}-l_{1}+(n-1) T_{1} n-2,3,4$. Tenemos $\Phi_{0}^{(3)} \mid-T_{1}^{6}$.

$\mathrm{Su}$ primera columna es:

$$
\tilde{\varphi}_{0}^{(3)}(u)= \begin{cases}\frac{1}{6}(u+3)(u+2)(u+1) & \text { for }-2 \leq u<-1, \\ -\frac{1}{2}(u+2)(u+1)(u-1) & \text { for }-1 \leq u<0, \\ \frac{1}{2}(u+1)(u-1)(u-2) & \text { for } 0 \leq u<1, \\ -\frac{1}{4}(u-1)(u-2)(u-3) & \text { for } 1 \leq u<2, \\ 0 & \text { otherwise. }\end{cases}
$$

y las siguientes son : $-F_{1} \dot{\bar{\varphi}}_{0}^{(3)}, F_{1}^{2} \ddot{\bar{\varphi}}_{0}^{(3)}, \mathrm{y}-F_{1}^{3} \ddot{\bar{\varphi}}_{0}^{(3)}$.

En general, el polinomio del $c$-ésimo intervalo de tiempo de izquierda a derecha del O-spline pasabajas de orden $K$ es dado por

$$
p_{c}(u)=\frac{1}{D_{c}} \prod_{\substack{n \\ n \neq c}}^{K+1}(u+n-c), \quad c=1,2, \ldots, K+1
$$

en donde la constante $D_{c}$ garantiza valor unitario en $u=0$ en cada polinomio. Se puede reconocer que el conjunto de polinomios en (16) coincide con el conjunto de pwolinomios de Lagrange de orden $K$ que interpolan una secuencia Dirac Kronecker en ventanas temporales de tamaño $K$ conteniendo su valor unitario central. Cada $\mathrm{O}$-spline es revestido con una pieza de cada uno de esos polinomios, formando el $K$-ésimo kernel de interpolación central de Lagrange. ${ }^{12} \mathrm{La}$ forma factorial en (16) permite el cálculo de cualquier O-spline, pieza por pieza, evitando el problema de singularidad encontrado en la inversión numérica de la matriz del operador de Taylor en (8) cuando $T_{1}$ es pequeño para cualquier orden $K \in \mathcal{I}$.

$\mathrm{En}^{12,22}$ y 23 se demuestra que cualquier pedazo del kernel de interpolación central de Lagrange converge al pedazo correspondiente de la función Sinc cuando $K \rightarrow \infty$
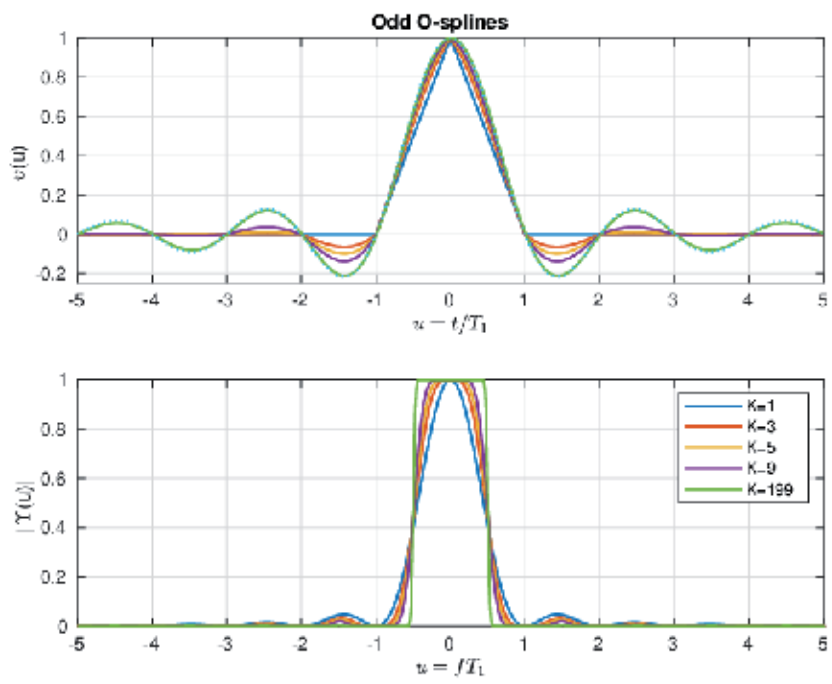

Fig. 1. O-splines pasabajas de orden impar y sus espectros abajo. 

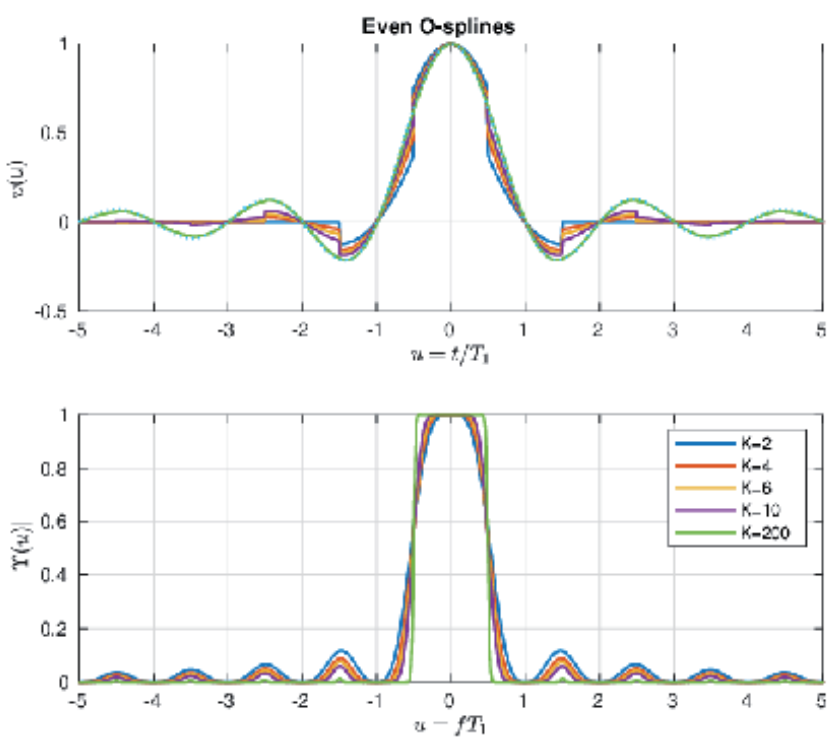

Fig. 2. O-splines pasabajas pares y sus espectros abajo.

\section{RESPUESTA EN FRECUENCIA DE LOS O-SPLINES}

El gráfico superior en la figura 1 ilustra algunos O-splines de orden impar, junto con la función Sinc $(\mathrm{K} \rightarrow \infty)$, y en la inferior sus espectros correspondientes. Como se puede ver, los O-splines forman respuestas impulsionales de filtros FIR que convergen al filtro ideal pasabajas. Cada trozo polinomial se aproxima al lóbulo correspondiente de la función Sinc. Sus respuestas en frecuencia tienen una ganancia pasabanda monotónica y la banda de paro tiene pequeños rizos, y en ese sentido son similares a las de los filtros IIR de Chebyshev tipo II. Los O-splines de orden par también convergen a la función Sinc pero con discontinuidades en los extremos de los trozos polinomiales, las cuáles producen rizos más altos en la banda de paro, como puede verse en los gráficos correspondientes de la figura 2.

La figura 3 muestra los espectros en $\mathrm{dB}$ de los $\mathrm{O}$-splines impares de la gráfica al fondo de la figura 1, para evaluar su rendimiento en análisis armónico. Para $\mathrm{K}=200$, la aproximación al filtro ideal es remarcable, ya que el primer lóbulo latera está en $-47 \mathrm{~dB}$, y las ganancias de paro a $-326 \mathrm{~dB}$, y para $\mathrm{K}=9$ están en -34 $\mathrm{dB}$ y $-350 \mathrm{~dB}$, respectivamente.

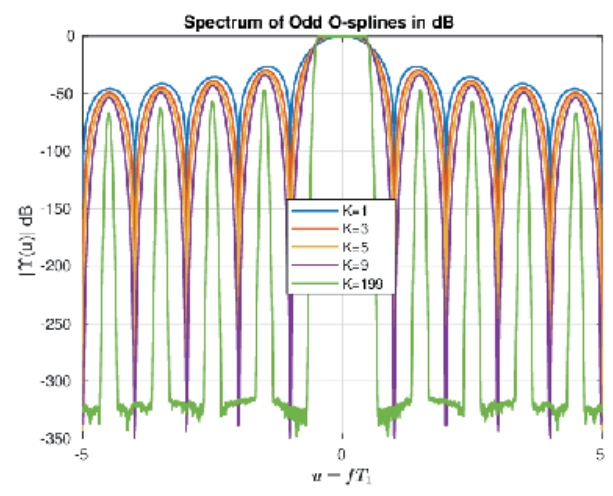

Fig. 3. Espectros en $d B$ de los O-splines pasabajas de orden impar. 
Finalmente, en la figura 4 se comparan los MK-splines cuadrático y cúbico con los O-splines de mismo soporte, el cúbico y quíntico. Se nota que ambos tipos tienen pasabanda plana, con la más ancha para el O-spline quíntico. El primer lóbulo lateral de los MK está a -32 y $-42 \mathrm{~dB}$, mientras que los de los $\mathrm{O}$ se encuentra a -30 y $-31.5 \mathrm{~dB}$, respectivamente. Sin embargo, los MK-splines no tienen bandas de paro máximamente lisas en las armónicas, mientras que los $\mathrm{O}-$ splines si las tienen. Medidas en la primera armónica las ganancias de los MKs son de -60 y $-64 \mathrm{~dB}$ y de -223 y $-322 \mathrm{~dB}$ para los $\mathrm{O}$-splines, respectivamente. Esta profesión es crucial en análisis armónico.

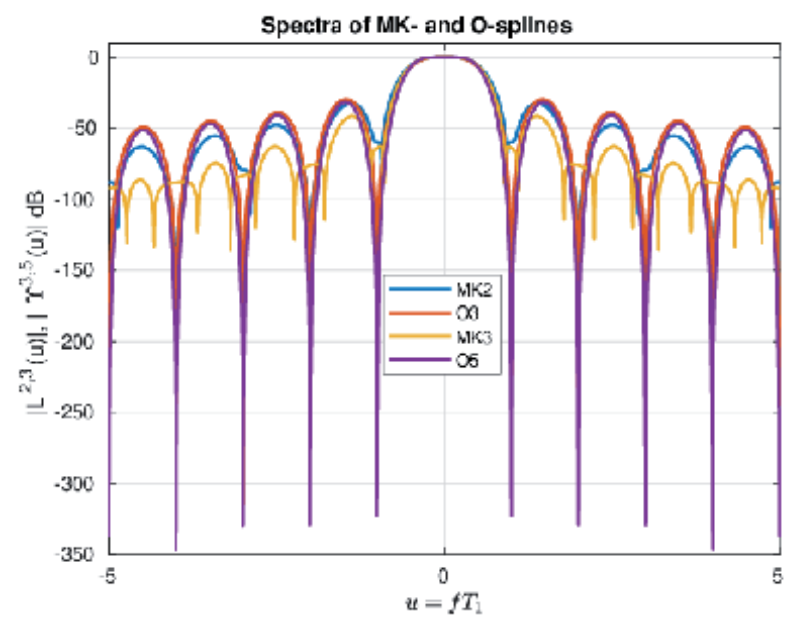

Fig. 4. MK-splines cuadrático y cúbico, comparados con el cubico y quíntico O-spline.

\section{O-SPLINE PASABAJAS Y SUS DERIVADAS}

Para un orden dado, la matriz dual contiene no solamente el O-spline pasabajas, sino también sus primeras derivadas. La figura 5 muestra en el primer renglón los primeros cuatro $\mathrm{O}$-splines y sus derivadas en las columnas correspondientes en orden creciente hacia abajo.

Al usar un conjunto vertical como respuestas impulsionales de un banco de filtros, éste ejecuta un muestreo (o remuestreo) de estados de la señal. Note que las derivadas sucesivas de un $\mathrm{O}$-spline son combinaciones lineales de traslaciones sucesivas de los $\mathrm{O}$-splines precedentes, formando una escalera de subespacios de los órdenes anteriores. La última derivada de la diagonal constituye el subespacio del orden más bajo V 0, generado con traslaciones de $\varphi 0(0)(\mathrm{t})$, los elementos de la primera supradiagonal están en el subespacio de primer orden V 1, el subespacio de funciones a trozos lineales, generado por traslaciones de $\varphi 0(1), y$ así sucesivamente, hasta el $\mathrm{O}$-spline de más alto grado.

Para el O-spline de tercer orden, se tienen las siguientes derivadas en términos de los O-splines de orden inferior. La primera derivada:

$$
\dot{\varphi}_{0}^{(3)}(u)=k_{1}\left[\varphi_{0}^{(2)}\left(u+\frac{1}{2}\right)-\varphi_{0}^{(2)}\left(u-\frac{1}{2}\right)\right]
$$

para la segunda:

$$
\left.\varphi_{0}^{(b)}(u)=F_{1}^{2} \cdot \varphi_{0}^{(1)}(u+1)-2 \varphi_{0}^{(1)}(u)+\varphi_{0}^{(1)}(u-1)\right] \text {. }
$$


y finalmente su tercera derivada:

$$
\begin{aligned}
\varphi_{01}^{(3)}(u)=F_{1}^{3} \varphi_{01}^{(0)}\left(u+\frac{3}{2}\right)-3 \varphi_{0}^{(0)}\left(u+\frac{1}{2}\right) \\
\left.+3 \varphi_{0}^{(0)}\left(u-\frac{1}{2}\right)-\varphi_{0}^{(0)}\left(u-\frac{3}{2}\right)\right] .
\end{aligned}
$$
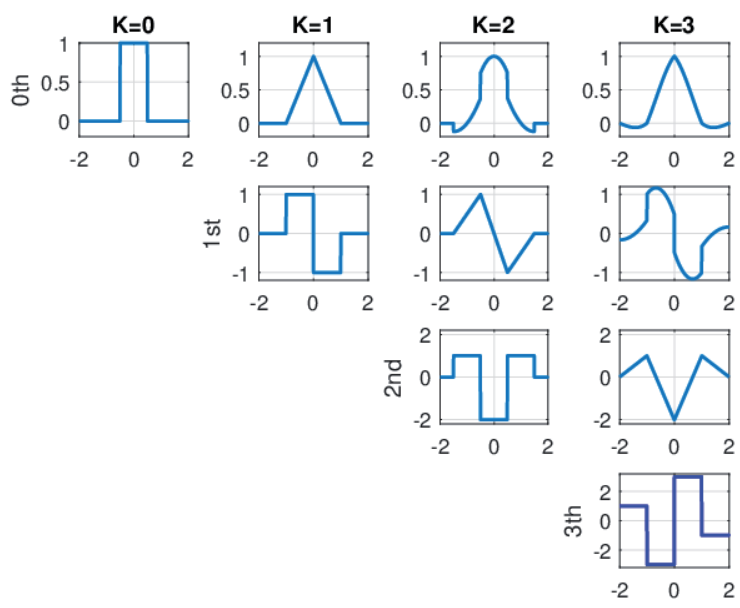

Fig. 5. En el renglón de arriba, los 0 -splines para $\mathrm{K}=0,1,2, \mathrm{y}, 3$, y bajo cada uno de ellos sus derivadas sucesivas.

\section{Respuesta impulsional y en frecuencia de los Diferenciadores}

El gráfico superior de la figura 6 muestra las respuestas impulsionales del primer diferenciador de orden 3 y $5,0(3)(\mathrm{u})$, y $0(5)$. Note que convergen a la primera derivada de la función Sinc mostrada con línea a rayas. mientras sus respuestas en frecuencia, en la gráfica inferior, convergen al del primer diferenciador pasabanda ideal. De hecho, si Hh(0)(f) es la respuesta en frecuencia del O-spline de orden $\mathrm{h}$, entonces la respuesta del primer diferenciador es $\mathrm{Hh}(1)(\mathrm{f})$ $=(\mathrm{j} 2 \pi \mathrm{f}) \mathrm{Hh}(0)(\mathrm{f})$, es decir, una ganancia lineal truncada por la respuesta en frecuencia del $\mathrm{O}$-spline de orden $\mathrm{h}$, como ilustra la línea a rayas.
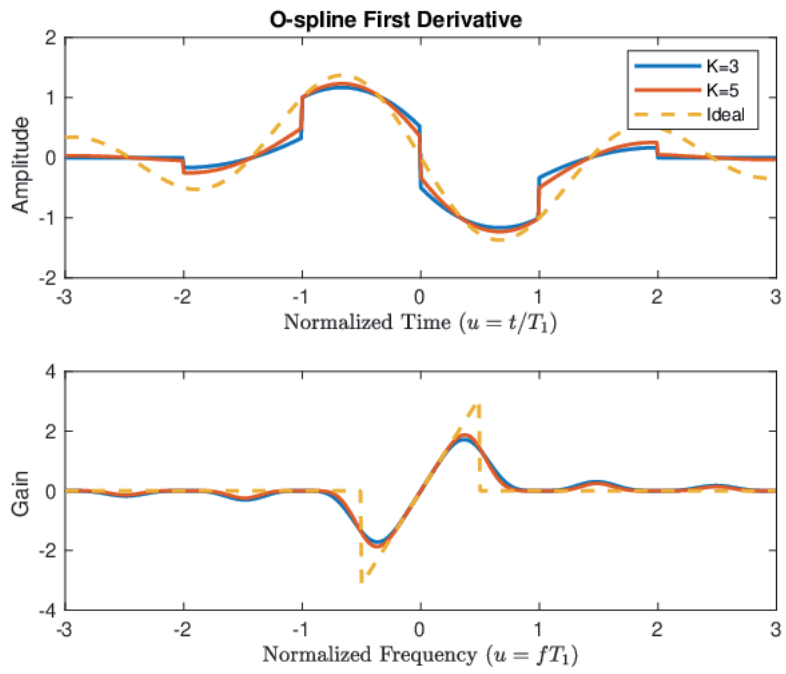

Fig. 6. Respuestas impulsional (arriba) y de frecuencia (abajo) del primer diferenciador con $K=3$ y $K=5$. Superpuestas las del ideal con líneas a rayas. 
Similarmente, las respuestas impulsionales de los segundos diferenciadores de orden 3 y 5 se muestran en la gráfica superior de la figura 7. Cuando el orden $\mathrm{K}$ $\rightarrow \infty$ convergen a la segunda derivada de la función Sinc; mientras sus respuestas en frecuencia, al fondo, tienen una ganancia parabólica en su banda de paso, truncada por la respuesta en frecuencia del O-spline correspondiente, ya que en este caso se tiene $\mathrm{Hh} 2(\mathrm{f})=(\mathrm{j} 2 \pi \mathrm{f}) 2 \mathrm{Hh} 0(\mathrm{f})$, una ganancia cuadrática ajustada a la ideal mostrada por la línea a rayas.
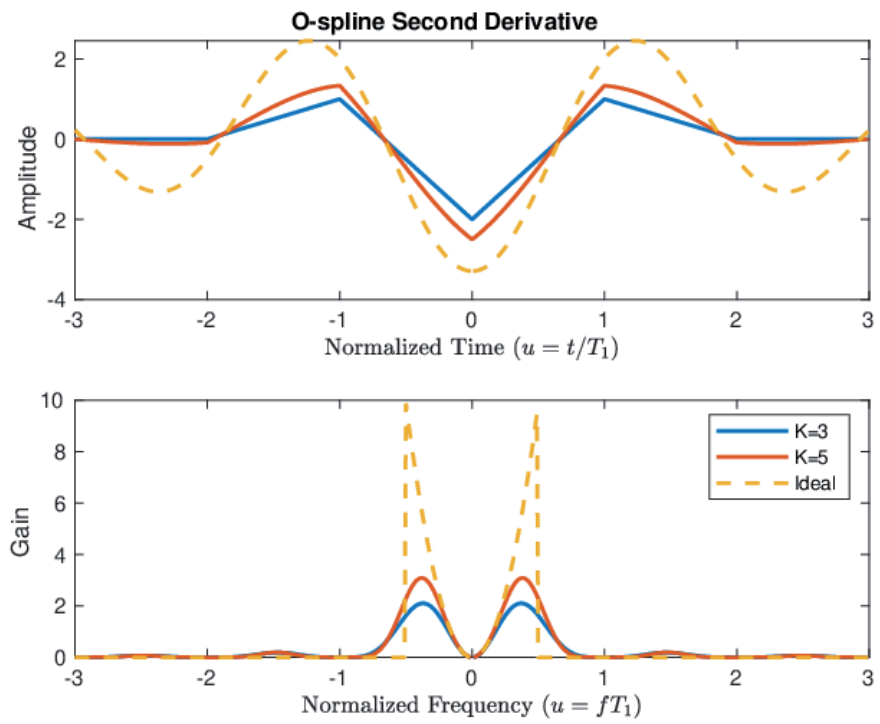

Fig. 7. Respuestas impulsional (arriba) y de frecuencia (abajo) del segundo diferenciador con $K=3$ y $K=5$. Superpuestas las del ideal con líneas a rayas.

\section{O-splines Armónicos}

Los O-splines armónicos son simplemente modulaciones de los O-splines pasabajas a la frecuencia de cada armónica:

$$
\widetilde{\varphi}_{h}^{(K)}(u)-\widetilde{\varphi}_{0}^{K}(u) e^{j 2 \pi h}, \quad h-0,1, \ldots, N-1 .
$$

Los cruces por cero de la función compleja exponencial coinciden con los de los O-splines pasabajas, preservando su propiedad cíclica.

\section{RESULTADOS}

En esta sección se usarán los O-splines de orden 3 como muestreadores de una señal senoidal de baja frecuencia, en la extracción de un modo de oscilación de frecuencia variable en un sistema eléctrico de potencia real, ${ }^{10}$ y para analizar los modos de oscilación de una forma de onda oscilográfica de presión arterial (BPOW, por sus siglas en Inglés) de un paciente de la base de datos presentada en. ${ }^{24}$ Señales reales con fuertes fluctuaciones de frecuencia son usadas para valorar el rendimiento del método propuesto en escenarios reales. Los O-splines se aplican cada ciclo fundamental, y se aplica interpolación de Taylor o de Hermite para reconstruir las señales. 


\section{SEÑALES MUESTREADAS E INTERPOLADAS}

La señal sinusoidal continua $\mathrm{s}(\mathrm{u})=\cos (\mathrm{U} / 4)$ y sus primeras dos derivadas se analizan y reconstruyen con el método propuesto. Como su espectro está localizado bajo las ganancias ideales de los diferenciadores, los errores de interpolación son pequeños. Esto ilustra el buen rendimiento de la técnica propuesta. La gráfica superior de la figura 8 muestra la señal y sus primeras dos derivadas mostrando con círculos las muestras estimadas. La desviación estándar de los errores de muestreo es de $4.2 \times 10-5,1.05 \times 10-5$, y $4.59 \times 10-4$ para la función, primera y segunda derivada, respectivamente. Los errores de interpolación obtenidos con esas muestras con interpolaciones de Taylor de orden 3,2 y 1 para la función, primera y segunda derivada se ilustran en la figura 9. Los errores máximos de dichas interpolaciones son de $6 \times 10-5,2.1 \times 10-4$, y 5 $\times 10-4$, respectivamente.
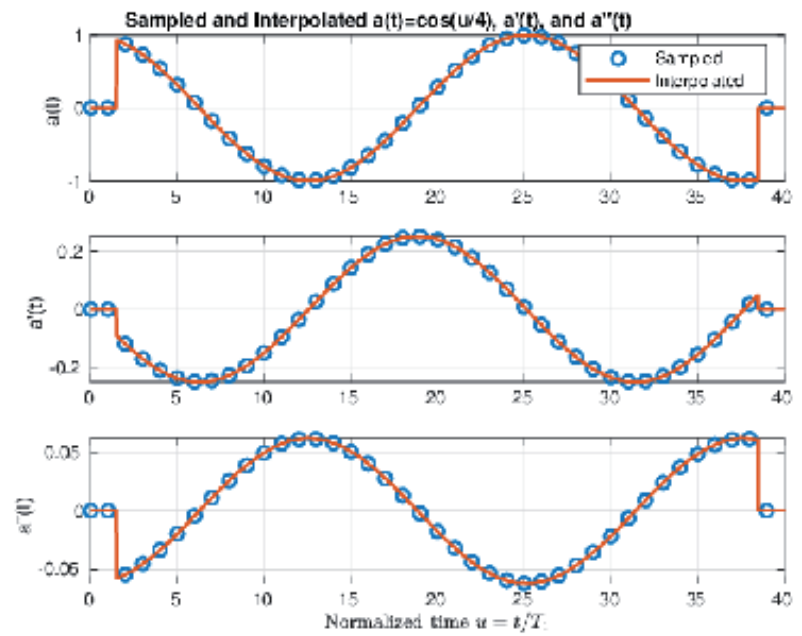

Fig. 8. Función Coseno muestreada e interpolada (arriba) y sus primeras dos derivadas abajo.
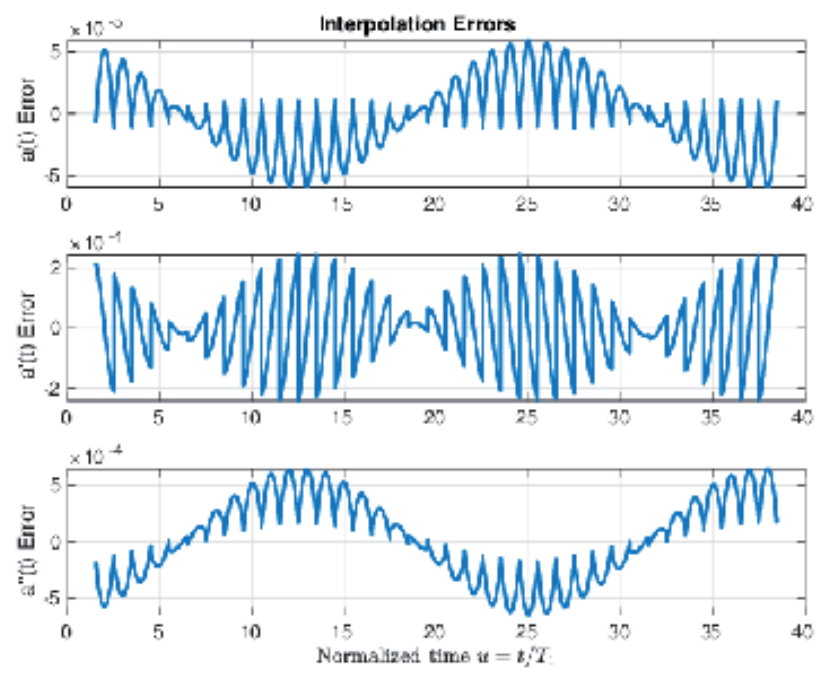

Fig. 9. Error de la señal sintetizada con el modelo de señal de Taylor, y errores de la primera y segunda derivada. 

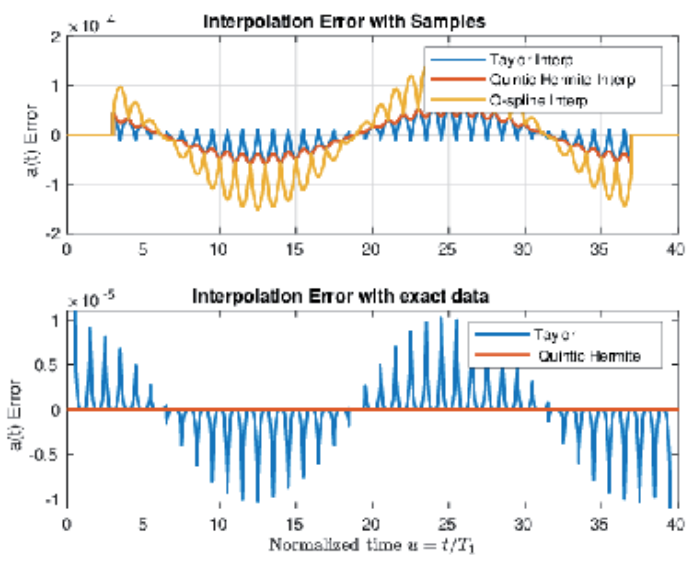

Fig. 10. Errores de interpolación con muestras (arriba), y con datos exactos (abajo).

Las muestras de la señal se interpolan con la interpolación de Taylor, el quinto esquema de Hermite (ver Apéndice B), con el O-spline (usado como interpolador), y con el spline cúbico de Keys. ${ }^{25}$ Los errores de interpolación se ilustran en el gráfico superior de la Fig. 10 comparados con el error de interpolación de Taylor. El error máximo más pequeño se obtiene con la interpolación de Taylor (con un error máximo de $\pm 6 \times 10-5$ ), enseguida, el error del interpolador quíntico de Hermite envuelve al error de la interpolación de Taylor, y seguido por el error de interpolación con el O-spline, con un error máximo de $1.4 \times 10-4$. El peor error de interpolación se obtiene con el spline cúbico de Keys, con un máximo error de $1.2 \times 10-2$, el cuál se encuentra fuera del rango de loa anteriores ilustrados en esa gráfica.

Para evaluar el rendimiento del método óptimo propuesto, en la gráfica inferior de la figura 10 se ilustra los errores obtenidos cuando las muestras exactas de la función y sus derivadas se usan para la interpolación de Taylor de orden 3 y la de Hermite de orden 5. Ambos errores, de Taylor y Hermite son nulos y planos junto a los polos. El error máximo de la interpolación de Taylor es de 10-5, mientras que el de Hermite es de $5 \times 10-9$. Comparando esta gráfica con la superior de la figura 9, se puede ver que el método propuesto alcanza un error solamente seis veces mayor que el teórico con interpolación cúbica. La precisión de las muestras estimadas aumenta con $\mathrm{O}$-splines de orden mayor.

\section{B. Oscilación de Potencia}

La oscilación de potencia analizada en ${ }^{10}$ es tomada como un segundo ejemplo. La oscilación se obtuvo de un sistema de voltajes y corrientes trifásicos, muestreados a 20 muestras por período fundamental en un sistema eléctrico de potencia de $50 \mathrm{~Hz}$, y se ilustra en la gráfica superior de la figura 11, con su espectro en la gráfica de en medio. El O-spline de orden 3 con una compresión temporal de dos, y un ancho de banda de $100 \mathrm{~Hz}$, cuyo espectro se ilustra en la gráfica del fondo, es usado para extraer la oscilación. $\mathrm{En}^{10}$ se demuestra que es un modo de oscilación modulado en frecuencia, yendo de cero a $15.6 \mathrm{~Hz}$, con un incremento exponencial. 

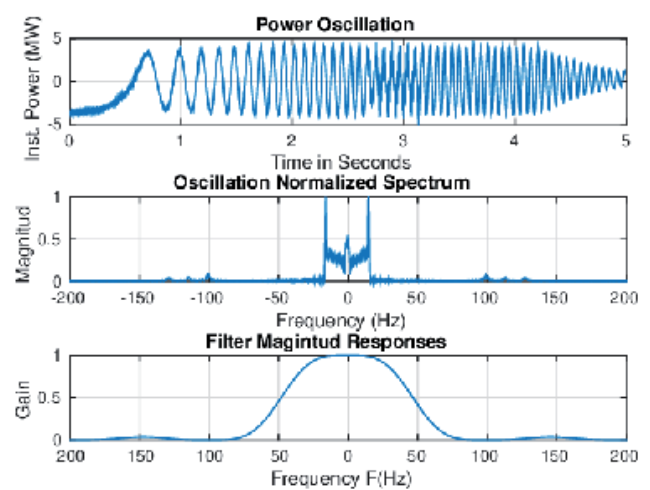

Fig. 11. Oscilación de potencia (arriba), su espectro (en medio), y la respuesta en frecuencia del filtro usado en el análisis (al fondo).
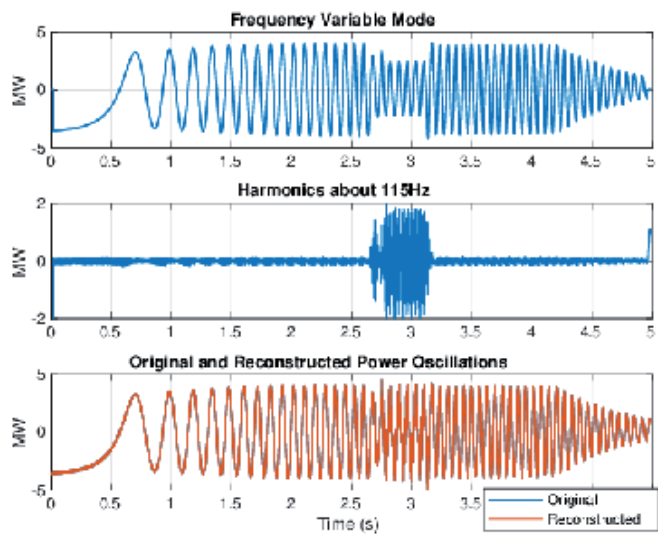

Fig. 12. Modo de frecuencia variable (arriba) y armónicas alrededor de $115 \mathrm{~Hz}$ (en medio), con la oscilación reconstruida y la original (abajo).

La señal es muestreada cada mitad de ciclo fundamental usando (5) e interpolación de Taylor es usada en esos intervalos con (3). El resultado se muestra en la figura 12. La gráfica superior muestra el modo de oscilación interpolado con polinomios de Taylor. Su frecuencia variable es perceptible porque s período de oscilación va disminuyendo muy rápido hacia el final de la ventana. El ruido en la gráfica de en medio es simplemente la diferencia entre la señal original y la interpolada. La gráfica del fondo muestra la superposición de la señal interpolada y el ruido, superpuesta sobre la original. Se nota que la reconstruida es casi perfecta, a pesar el importante ruido localizado durante la falla. La varianza de la diferencia entre la oscilación interpolada de Taylor y sus estimados instantáneos obtenidos con el muestreador de estados O-spline es igual a 0.0127, el cuál es inferior al $0.32 \%$ de la máxima amplitud de la oscilación.

\section{Forma de Onda Oscilométricas de Presión Arterial}

La estimación de presión sistólica y diastólica de datos numéricos de $\mathrm{BPOWs}^{24}$ es un reto interesante debido a la estructura compleja de estas señales. La extracción del modo fundamental es crucial ya que éste muestra la pulsación principal del corazón. Ambas presiones son estimadas de la envolvente de amplitud de este modo de oscilación. 


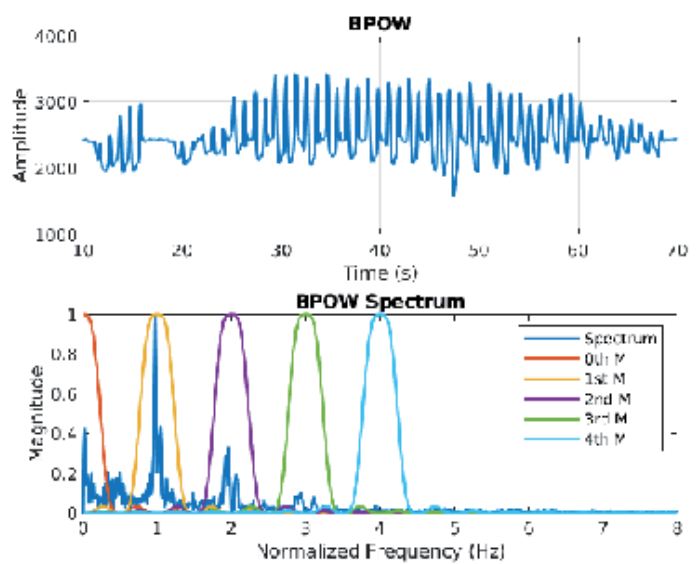

Fig. 13. BPOW (arriba), su espectro y las respuestas en frecuencia de los filtros de análisis (al fondo).

Los demás modos son debidos al comportamiento no linealidad de la manga.

La gráfica superior de la figura 13 muestra la señal BPOW analizada, muestreada a 150 muestras por segundo. Su estructura básica temporal consiste de picos repetitivos con formas de parábolas colgantes que brincan hacia arriba y hacia abajo. Su espectro es ilustrado en la gráfica de abajo junto con las respuestas en frecuencia de los O-splines armónicos usados en la descomposición. Éstos se obtienen modulando el O-spline pasabajas de orden 3 (mbox III-D mbox ) dilatado al doble (a 8 ciclos) para reducir su ancho de banda a la mitad. Las muestras se obtienen aplicando producto punto a la señal con traslaciones sucesivas de los O-splines armónicos y sus derivadas, y los modos se reconstruyen con los estimados instantáneos en de (5).

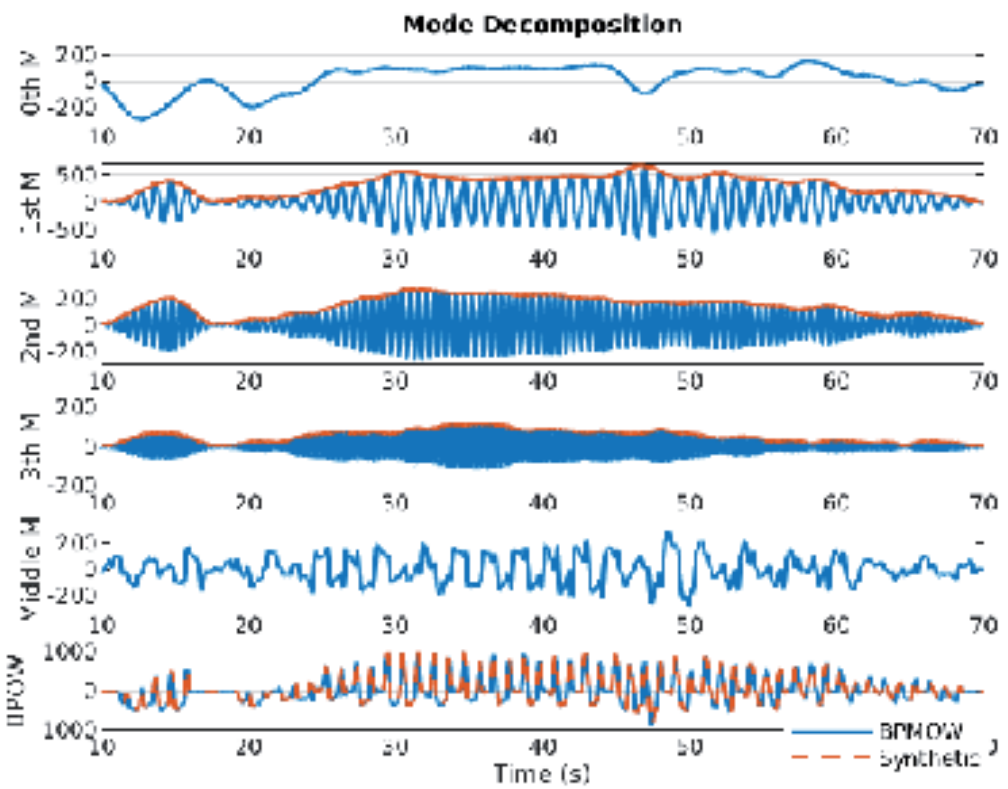

Fig. 14. Descomposición oscilográfica de la BPOW. De arriba hacia abajo los modos predominantes $(0,1,2$, y 3$)$ seguida pr la señal con frecuencias interarmónicas. Al fondo, la señal sintética superpuesta a la original con reconstrucción casi perfecta. 
Los modos predominantes de la BPOW se ilustran en las cuatro gráficas superiores de la figura 14, seguida por la señal residual, y finalmente, en la gráfica del fondo, la señal sintética estimada comparada con la señal original. Note que el modo de oscilación de orden cero no es constante, sino que fluctúa a muy baja frecuencia. La envolvente del primer modo (línea roja de la segunda gráfica) tiene la forma parabólica esperada. Por otra parte la señal residual (formada por la energía espectral en las bandas de paro de los filtros) tiene forma de cigüeñal que explica los brincos de las formas parabólicas en la gráfica superior de la figura 13.

La tabla I muestra el error cuadrático medio (MSE) como porciento de la energía de la BPOW cuando los modos consecutivos se van agregando en la señal reconstruida, formando reconstrucción perfecta cuando se incluyen todas las componentes. Las discrepancias se deben a la complejidad de las fluctuaciones de la señal BPOW, cuyos modos no están perfectamente delimitados, como se puede ver en la gráfica del fondo de la figura 13.

Tabla I. Error cuadrático medio normalizado al ir incluyendo modos consecutivos.

\begin{tabular}{|c|c|c|c|c|c|}
\hline \multicolumn{6}{|c|}{ Error Cuadrático Medio (\%) } \\
\hline 0 & 1 ero & 2 do & 3 ero & 40 & Residual \\
87.64 & 31.22 & 19.08 & 17.19 & 17.00 & 0.00 \\
\hline
\end{tabular}

\section{DISCUSIÓN}

El kernel de interpolación central de Lagrange se usa en la literatura como interpolador, ${ }^{22,14} \mathrm{o}$ como un kernel de aproximación a la función Sinc, ${ }^{12}$ pero no como un kernel de análisis, y mucho menos como un muestreador de los estados de una señal. Esta es la contribución de este artículo, junto con la derivación en forma cerrada de las funciones de los O-splines, la cual reduce la complejidad computacional de la DTTFT, especialmente cuando la señal contiene armónicas, como los casos ilustrados en la sección anterior.

$\mathrm{En}^{12}$ se compara el rendimiento del kernel interpolador central de Lagrange y el spline cardenal fundamenta $1^{6}$ de órdenes sucesivos. Sin embargo, esta comparación es inválida en nuestra aplicación de análisis, ya que los splines fundamentales tienen soporte infinito, mientras que el soporte de los kerneles de Lagrange son finitos.

El método propuesto difiere también del propuesto en, ${ }^{13}$ ya que la función dual de la base interpolatoria no tiene soporte finito. También difiere del usado en, ${ }^{10}$ en el cuál únicamente la ecuación de análisis (5) es utilizada con el O-spline y sus derivadas. En el aquí propuesto se usa también la ecuación de síntesis en la interpolación.

\section{CONCLUSIONES}

Los O-splines son las respuestas impulsionales de los filtros pasabanda de la DTTFT. Son modulaciones a las frecuencias armónicas del filtro pasabajas. Ellos actúan como el filtro ideal pasabajas con ganancias ideales de diferenciación en las frecuencias armónicas y se usan como muestreadores de los estados de señales 
oscilatorias de banda limitada, ofreciendo un algoritmo de compresión de datos óptimo para modos oscilatorios, con un error suficientemente pequeño graduado por el orden del O-spline. Son muy útiles para captar oscilaciones de las cuáles no sólo interesa estimar o interpolar la pura señal, sino también sus derivadas, ofreciendo un potente estimador de estados implementado por filtros FIR, con un gran potencial para aplicaciones de pronóstico y control de sistemas dinámicos.

\section{Apéndice A}

Determinante de Matriz Vandermonde

Dada la matriz de Vandermonde:

$$
V_{K}=\left(\begin{array}{ccccc}
1 & c_{1} & c_{1}^{2} & \ldots & c_{1}^{K-1} \\
1 & c_{2} & c_{2}^{2} & \ldots & c_{2}^{K-1} \\
\vdots & \vdots & \vdots & \ddots & \vdots \\
1 & c_{K} & c_{K}^{2} & \ldots & c_{K}^{K-1}
\end{array}\right)
$$

se sabe que:

$$
\operatorname{det}\left(V_{K}\right)=\left[\prod_{k-1}^{K-1}\left(c_{K}-c_{K-k}\right)\right] \operatorname{det}\left(V_{K-1}\right) .
$$

En el caso de $\Phi_{0}^{(K)}, t_{K}-t_{K-k}=k T, \mathrm{y}$

$$
\operatorname{det}\left(\Phi_{0}^{(K)}\right)=T^{K} \operatorname{det}\left(\Phi_{0}^{(K-1)}\right)
$$

como los factoriales de los términos de Taylor en las columnas de columns de $\Phi_{0}^{(K)}$ cancelan los factoriales del determinante de la matriz Vandermonde. Por tanto:

$$
\operatorname{det}\left(\Phi_{0}^{(K)}\right)=T^{K} T^{K-1} T^{K-2} \cdots T^{2} T^{\prime}=T^{\frac{K i K+1}{2}} .
$$

con $\operatorname{det}\left(\Phi_{0}^{(0)}\right)=1$.

\section{Apéndice B}

Interpolador Quíntico de Hermite

La función $f(u)$ entre los instantes $t_{0}$ y $t_{1}$, con estados $\left(y_{0}, \dot{y}_{0}, \ddot{y}_{0}\right)$ y $\left(y_{1}, \dot{y}_{1}, \ddot{y}_{1}\right)$ es la siguiente:

$$
\begin{aligned}
& f(u)=y_{0} \varphi_{0}(u)+\dot{y}_{0} \varphi_{1}(u)+\ddot{y}_{0} \varphi_{2}(u) \\
& +y_{1} \varphi_{3}(u)+\dot{y}_{1} \varphi_{4}(u)+\ddot{y}_{1} \varphi_{5}(u)
\end{aligned}
$$

con los siguientes polinomios quínticos:

$$
\begin{aligned}
& \varphi_{0}(u)=1-10 u^{3}+15 u^{4}-6 u^{5} \\
& \varphi_{1}(u)=u-6 u .^{3}+8 u^{4}-3 u^{5} \\
& \varphi_{2}(u)=\left(u^{2}-3 u^{3}+3 u^{4}-u 5\right) / 2 \\
& \varphi_{3}(u)=10 u^{3}-15 * u^{4}+6 u^{5} \\
& \varphi_{4}(u)=-4 u^{3}+7 u^{4}-3 * u^{5} \\
& \varphi_{5}(u)=\left(u^{3}-2 * u^{4}+u^{5}\right) / 2 .
\end{aligned}
$$




\section{REREFERNCIAS}

1. C. de Boor, A practical Guide to Splines. Springer, 2001.

2. I. J. Schoenberg, Cardinal Spline Interpolation. SIAM, 1973.

3. D. Pang, L. A. Ferrari, and P. Sankar, "B-spline FIR filters," Circuits Systems and Signal Process, vol. 13, no. 1, pp. 31-64, 1994, available: https://doi. org/10.1007/BF01183840.

4. T. M. Lehmann, C. Gonner, and K. Spitzer, "Survey: interpolation methods in medical image processing," IEEE Trans. Med. Imag., vol. 18, no. 11, pp. 1049-1075, Nov 1999.

5. S. G. Mallat, "A theory for multiresolution signal decomposition: the wavelet representation," IEEE Trans. Pattern Anal. Mach. Intell., vol. 11, no. 7, pp. 674-693, July 1989.

6. M. Unser, A. Aldroubi, and M. Eden, "B-spline signal processing. Part I- theory," IEEE Trans. Signal Process., vol. 41, no. 2, pp. 821-833, Feb 1993.

7. _ , "B-spline signal processing. Part II - efficiency design and applications," IEEE Trans. Signal Process., vol. 41, no. 2, pp. 834-848, Feb 1993.

8. M. Unser and T. Blu, "Cardinal exponential splines: part I - theory and filtering algorithms," IEEE Trans. Signal Process., vol. 53, no. 4, pp. 1425-1438, April 2005.

9. M. Unser, "Cardinal exponential splines: part II - think analog, act digital," IEEE Trans. Signal Process., vol. 53, no. 4, pp. 1439-1449, April 2005.

10.J. A. de la O Serna, "Analyzing power oscillating signals with the O-splines of the Discrete Taylor-Fourier Transform," IEEE Trans. Power Syst., vol. 33, no. 6, pp. 7087-7095, Nov 2018.

11.J. A. de la O Serna, M. R. A. Paternina, A. Zamora-Méndez, R. K. Tripathy, and R. B. Pachori, "EEG-rhythm specific taylor-fourier filter bank implemented with O-splines for the detection of epilepsy using EEG signals," IEEE Sensors J., pp. 1-1, 2020.

12.E. H. W. Meijering, W. J. Niessen, and M. A. Viergever, "The Sincapproximating kernels of classical polynomial interpolation," in IEEE International Conference on Image Processing (ICIP'99), vol. 3, Oct 1999, pp. 652-656.

13.M. Unser and A. Aldroubi, "A general sampling theory for nonideal acquisition devices,” IEEE Trans. Signal Process., vol. 42, no. 11, pp. 2915-2925, Nov 1994.

14.E. Meijering, "A chronology of interpolation: from ancient astronomy to modern signal and image processing," Proceedings of the IEEE, vol. 90, no. 3, pp. 319-342, March 2002.

15.J. Chen and Z. Cai, "Cardinal MK-spline signal processing: Spatial interpolation and frequency domain filtering," Information Sciences, vol. 495, pp. 116 - 135, 2019. [Online]. Available: http://www.sciencedirect. com/science/article/pii/S0020025519303767

16.J. Chen and Z. Cai, "A new class of explicit interpolatory splines and related measurement estimation,” IEEE Trans. Signal Process., pp. 1-1, 2020. 
17.G. Rogers, Power System Oscillations. Boston: Springer, 2000.

18.A. Roman Messina, Inter-area Oscillations in Power Systems: A Nonlinear and Nonstationary Perspective. Springer, 2009.

19.M. Forouzanfar, H. R. Dajani, V. Z. Groza, M. Bolic, S. Rajan, and I. Batkin, "Oscillometric blood pressure estimation: Past, present, and future," IEEE Reviews in Biomedical Engineering, vol. 8, pp. 44-63, 2015.

20.J. G. Proakis and M. Salehi, Digital Communications, 5th ed. Boston, MA: McGraw Hill, 2008, sec. 2.1.

21.M. Vetterli, K. Kovacevic, and V. K. Goyal, Foundations of Signal Processing, 3rd ed. Cambridge, UK: Cambridge University Press, 2014, available at http://www.fourierandwavelets.org/.

22.H. W. Meijering, "Image enhancement in digital x-ray angiography." [Online]. Available: https://dspace.library.uu.nl/bitstream/handle/1874/367/c6.pdf

23.A. K. Jain, Fundamentals of Digital Image Processing. Prentice-Hall,Englewood Cliffs, New Jersey,USA, 1989.

24.J. A. De la O Serna, "Taylor-Fourier analysis of blood pressure oscillometric waveforms," IEEE Trans. Instrum. Meas., vol. 62, no. 9, pp. 2511-2518, 2013. [Online]. Available: http://dx.doi.org/10.1109/TIM.2013.2258245

25.R. Keys, "Cubic convolution interpolation for digital image processing," IEEE Trans. Signal Process., vol. 29, no. 6, pp. 1153-1160, December 1981. 